\title{
Normalized Cut Based Edge Detection
}

\author{
Mario Barrientos and Humberto Madrid \\ Applied Mathematics Research Center \\ Autonomous University of Coahuila \\ Camporedondo Unit, Building "S", Postcode 25000 \\ Saltillo, Coahuila, Mexico \\ \{mbarrientosmate, hmadrid\}@gmail.com \\ wWw.cima.uadec.mx
}

\begin{abstract}
This work introduces a new technique for edge detection based on a graph theory tool known as normalized cut. The problem involves to find certain eigenvector of a matrix called normalized laplacian, which is constructed in such way that it represents the relation of color and distance between the image's pixels. The matrix dimensions and the fact that it is dense represents a trouble for the common eigensolvers. The power method seemed a good option to tackle this problem. The first results were not very impressive, but a modification of the function that relates the image pixels lead us to a more convenient laplacian structure and to a segmentation result known as edge detection. A deeper analysis showed that this procedure does not even need of the power method, because the eigenvector that defines the segmentation can be obtained with a closed form.
\end{abstract}

Keywords: Edge detection, normalized cut, power method, image segmentation.

\section{Normalized Cut}

A graph $G$ its conformed by a pair of sets $(V, E)$, where $V$ is a finite set of points $v_{1}, v_{2}, \ldots, v_{n}$ called nodes, and $E$ is the set of edges $e(i, j)$ that connects the nodes $v_{i}$ and $v_{j}$. This edges will have an assigned weight $w_{i j}$ and will be non directed, which means that $w_{i j}=w_{j i}$. The idea to construct a graph from an image is consider each pixel of the image as a node of the graph. The weights of the edges will be assigned with a function that relates pairs of pixels, taking into account characteristics like the color similarity and the distance among them.

A graph $G=(V, E)$ can be splitted in two disjoint sets $A$ and $B$, with $A \cup B=$ $V$ y $A \cap B=\emptyset$, simply by removing the edges connecting both parts. We say that $A$ and $B$ are a bipartition of $G$. Their grade of dissimilarity can be calculated as the sum of the weights of the removed edges. In graph theory this process is called cut:

$$
\operatorname{cut}(A, B)=\sum_{u \in A, v \in B} w(u, v)
$$


with $w(i, j)=w_{i j}$. The optimal bipartition is the one that minimizes the cut value. Wu and Leahy [1] proposed a grouping method based on the minimum cut criteria that produced good segmentations for some images. They also noted that the minimum cut criteria favors the formation of small sets containing only a few isolated nodes. To avoid this tendency, Shi and Malik 2] proposed a new measure of dissociation of groups called Normalized Cut (Ncut)

$$
\operatorname{Ncut}(A, B)=\frac{\operatorname{cut}(A, B)}{\operatorname{assoc}(A, V)}+\frac{\operatorname{cut}(A, B)}{\operatorname{assoc}(B, V)},
$$

where $\operatorname{assoc}(A, V)=\sum_{u \in A, t \in V} w(u, t)$ is the total of connections of the nodes from $A$ to the nodes of the graphic and $\operatorname{assoc}(B, V)$ is defined similarly.

\subsection{Calculating the Optimal Partition}

Given a partition of the nodes of a graph $G$ in two sets $A$ and $B$, we make:

- $\mathbf{x}$ an indicator vector of dimension $N=|V|$, with $\mathbf{x}_{i}=1$ if the node $i$ is in $A$ and $\mathbf{x}_{i}=-1$ if the node belongs to $B$.

- W a symmetric matrix of $N \times N$ with $\mathbf{W}(i, j)=w_{i j}$, known as adjacency matrix.

- D a diagonal matrix of $N \times N$ whose diagonal elements are the row-wise sum of the entries of $\mathbf{W}, \mathbf{D}(i, i)=\sum_{j} \mathbf{W}(i, j)$.

In 2 is shown that minimize (2) is equivalent to solve

$$
\min _{x} \operatorname{Ncut}(\mathbf{x})=\min _{y} \frac{\mathbf{y}^{T}(\mathbf{D}-\mathbf{W}) \mathbf{y}}{\mathbf{y}^{T} \mathbf{D} \mathbf{y}}
$$

with $\mathbf{1}$ a vector of all-one elements of dimension $N$ such that $\mathbf{y}^{T} \mathbf{D} \mathbf{1}=0$ and $\mathbf{y}_{i} \in\{1,-b\}$, where $b$ depends of the proportion of the cardinalities of $A$ and $B$. The procedure to obtain (3) and how $b$ is defined can be found in 3 .

Note that (3) is a Rayleigh quotient [4. If the domain restriction of $\mathbf{y}$ is relaxed and it is allowed to take real values we can minimize Ncut solving the eigensystem

$$
(\mathbf{D}-\mathbf{W}) \mathbf{y}=\lambda \mathbf{D} \mathbf{y}
$$

that can be rewrited as the standard eigensystem

$$
\mathbf{D}^{-\frac{1}{2}}(\mathbf{D}-\mathbf{W}) \mathbf{D}^{-\frac{1}{2}} \mathbf{z}=\lambda \mathbf{z},
$$

with $\mathbf{z}=\mathbf{D}^{\frac{1}{2}} \mathbf{y}$.

Is easy to verify that $\mathbf{z}_{0}=\mathbf{D}^{\frac{1}{2}}$ is the eigenvector of (5) with associated eigenvalue zero. Moreover, $\mathbf{D}^{-\frac{1}{2}}(\mathbf{D}-\mathbf{W}) \mathbf{D}^{-\frac{1}{2}}$, known as normalized Laplacian, is symmetric positive-semidefinite because is known that $(\mathbf{D}-\mathbf{W})$ is positivedefinite [5]. Therefore $\mathbf{z}_{0}$ is the eigenvector associated to the smallest eigenvalue, also called smallest eigenvector of (5) and all of the eigenvectors of (5) are perpendicular to each other. Retaking one of the main characteristics of the Rayleigh 
quotient, the second smallest eigenvector $\mathbf{z}_{1}$ of the generalized eigensystem (44) is the real-valued solution of the normalized cut problem [4].

In [3] is shown that the bipartion defined by the signs of the elements of $\mathbf{y}$ and $\mathbf{z}_{1}$ is the same. This property is not necessarily preserved numerically. To deal with this, some criterion should be adopted to split $\mathbf{z}_{1}$ in two sets.

\section{Power Method}

The practical difficulty of segmentation using the normalized cut method lies in the enormous amount of data that needs to be generated and stored. An image of $m \times n$ generates a Laplacian of $N \times N$, with $N=m \cdot n$. The Lanczos method has been used formerly to find $\mathbf{z}_{1}$ [2. We proposed to use power method to get this vector, because this method has shown good performance in other problems involving big dimensions matrices, such as PageRank [6].

The power method is enounced as follows:

Let $\mathbf{A}$ be a $n \times n$ diagonalizable matrix with eigenvalues $\lambda_{1}, \lambda_{2}, \ldots, \lambda_{n}$, such that $\left\|\lambda_{1}\right\|>\left\|\lambda_{j}\right\|$ for $j=2, \ldots, n$ ( $\lambda_{1}$ is the dominant eigenvalue), and $\mathbf{x}_{0}$ an initial vector of dimension $n$. The sequence

$$
\mathbf{x}_{k}=\mathbf{A} \mathbf{x}_{k-1}
$$

converge to the eigenvector $\mathbf{v}_{1}$ associated to $\lambda_{1}\left(\mathbf{v}_{1}\right.$ is the dominant eigenvector).

The initial vector $\mathbf{x}_{0}$ can be chosen randomly. The stop criterion we used is the relative error between the iterations $k$ and $k-1$.

The eigenvector that we need to obtain the segmentation is not the dominant one, so we need to use deflation and shift [4] to obtain it with the power method. We know that the normalized laplacian $\mathbf{L}$ is symmetric positive semi-definite, so its smallest eigenvector is $\mathbf{u}_{1}=\mathbf{D}^{\frac{1}{2}} \mathbf{1}$ with $\lambda_{1}=0$, and that its eigenvalues are on the interval $[0,2]$, [] . Considering this, the procedure to calculate the subdominant eigenvector of $\mathbf{L}$ is:

1. Apply a shift to $\mathbf{L}$ with $s=2$ to obtain $\mathbf{L}_{s}=\mathbf{L}-s \mathbf{I}$, with $\mathbf{I}$ identity matrix of $N \times N$.

2. Deflate $\mathbf{L}_{s}$ using $\overline{\mathbf{u}}_{1}=\mathbf{u}_{1} /\left\|\mathbf{u}_{1}\right\|$ and $\lambda_{1}^{\prime}=-2$, obtaining $\mathbf{L}_{d}=\mathbf{L}_{s}-\lambda_{1}^{\prime} \overline{\mathbf{u}}_{1} \overline{\mathbf{u}}_{1}^{T}$.

3. Use power method with $\mathbf{L}_{d}$ to get $\mathbf{u}_{2}$.

To avoid the explicit construction of $\overline{\mathbf{u}}_{1} \overline{\mathbf{u}}_{1}^{T}$ required for the deflation, we will do the next modification to the procedure. After shift and deflation the matrix $\mathbf{L}$ becomes

$$
\mathbf{L}_{d}=\mathbf{L}_{s}-\lambda_{1}^{\prime} \overline{\mathbf{u}}_{1} \overline{\mathbf{u}}_{1}^{T} .
$$

Multiplying (6) by $\mathbf{x}_{0}$ produces

$$
\begin{aligned}
\mathbf{L}_{d} \mathbf{x}_{0} & =\left(\mathbf{L}_{s}-\lambda_{1}^{\prime} \overline{\mathbf{u}}_{1} \overline{\mathbf{u}}_{1}^{T}\right) \mathbf{x}_{0} \\
& =\mathbf{L}_{s} \mathbf{x}_{0}-\lambda_{1}^{\prime} \overline{\mathbf{u}}_{1}\left(\overline{\mathbf{u}}_{1}^{T} \mathbf{x}_{0}\right) .
\end{aligned}
$$


We make $\mathbf{x}_{1}=\mathbf{L}_{d} \mathbf{x}_{0}$. Now the product of $\mathbf{L}_{d} \mathbf{x}_{1}$ is

$$
\mathbf{L}_{d} \mathbf{x}_{1}=\mathbf{L}_{d}^{2} \mathbf{x}_{0}=\mathbf{L}_{s} \mathbf{x}_{1}-\lambda_{1}^{\prime} \overline{\mathbf{u}}_{1}\left(\overline{\mathbf{u}}_{1}^{T} \mathbf{x}_{1}\right)
$$

Continuing in the same way $\mathbf{x}_{k}$ will be

$$
\mathbf{x}_{k}=\mathbf{L}_{d}^{k} \mathbf{x}_{0}=\mathbf{L}_{s} \mathbf{x}_{k-1}-\lambda_{1}^{\prime} \overline{\mathbf{u}}_{1}\left(\overline{\mathbf{u}}_{1}^{T} \mathbf{x}_{k-1}\right)
$$

Hence, we will do the power method iterations as is indicated by (7).

\section{Weight Function and Segmentation}

A crucial point for the segmentation result is the election of the weight function with which the edges weights will be assigned. In [2] the proposed function is:

$$
w_{i j}=e^{\frac{-\|F(i)-F(j)\|^{2}}{\sigma_{I}}} *\left\{\begin{array}{l}
e^{\frac{-\|X(i)-X(j)\|^{2}}{\sigma}} \text { if }\|X(i)-X(j)\| \leq r \\
0 \text { otherwise }
\end{array}\right.
$$

$F$ represents the color characteristics of the pixels. For an RGB image $F(i)=$ $(r(i), g(i), b(i))$, considering that the pixels of the image are reshaped as an $N$ size vector and being $r(i), g(i)$ and $b(i)$ the corresponding values for the pixel $i$ in the red, green and blue layers; for images on HSV format $F$ will be $F(i)=[v(i), v \cdot s \cdot \sin (2 \pi \cdot h), v \cdot s \cdot \cos (2 \pi \cdot h)]$, being $h(i), s(i)$ and $v(i)$ the corresponding hue, saturation and value components of each pixel; finally, $F$ will be the intensity of the pixel for a grayscale image. For all the cases, $X(i)$ will be the location of the pixel on the image, being $(1,1)$ the upper left corner. The $r$ parameter will define a neighborhood for each pixel. It is considered that the pixels that are further than $r$ have not significative relationship with the central pixel. $\sigma_{I}$ and $\sigma_{X}$ are parameters that need to be calibrated.

Once the weight function has been set, we only need to define a criterion to split $\mathbf{u}_{2}$ in two sets, a threshold in this case, to finally have the complete segmentation process defined. As is supported in [3], the best results are obtained splitting the elements according to its sign, that is

$$
\mathbf{x}(i)=\left\{\begin{aligned}
1 & \text { if } \mathbf{u}_{2}(i)>0 \\
-1 & \text { if } \mathbf{u}_{2}(i) \leq 0
\end{aligned}\right.
$$

The results obtained using power method and the weight function (8) were good, but the memory and computing time requirements were not improved significantly, as can be seen in [8]. 


\section{Edge Detection}

Our first modification to the segmentation procedure was the introduction of the weight function

$$
w_{i j}=e^{\frac{-\|F(i)-F(j)\|}{\sigma} I} *\left\{\begin{array}{l}
e^{\frac{-\|X(i)-X(j)\|}{\sigma} X} \\
\text { if }\|X(i)-X(j)\| \leq 1 \\
0 \text { otherwise }
\end{array} .\right.
$$

The differences with (8) are that the arguments of the exponential functions are not squared, and that $r$ is fixed to 1 , this last being the most important one. The segmentation obtained with a grayscale image using (10) is presented on Fig. 1
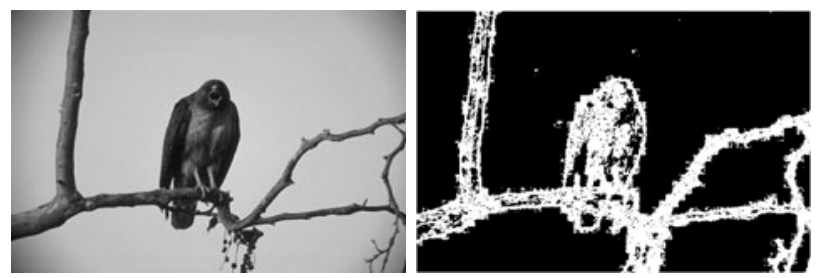

Fig. 1. First example of edge detection using a $354 \times 451$ pixels grayscale image with $\sigma_{I}=255$ and $\sigma_{X}=\sqrt{354^{2}+451^{2}}$

This kind of segmentation is known as edge detection. The distance factor does not modify the resulting segmentation, so we can drop it and define our weight function for edge detection as

$$
w_{i j}=\left\{\begin{array}{l}
e^{\frac{-\|F(i)-F(j)\|}{\sigma_{I}}} \text { if }\|X(i)-X(j)\| \leq 1 \\
0 \text { otherwise }
\end{array} .\right.
$$

The segmentation results are greatly improved by applying a median filter to the image. This filter has the property of remove noise preserving the edges 9 . For images on RGB and HSV formats, the filter is applied to each layer of the image. The obtained edges are thick, but they can be thinned using methods like non maxima suppression [10] or the edge thinning algorithm implemented in the MATLAB's Image Processing Toolbox [11, if necessary. To show the segmentation results at several $\sigma_{I}$ values, the results are presented as soft boundary maps in Fig. 2

\subsection{Simplifying the Method}

An interesting fact of this edge detection method is that the power method always needs only one iteration to converge with $1 \times 10^{-5}$ precision when 

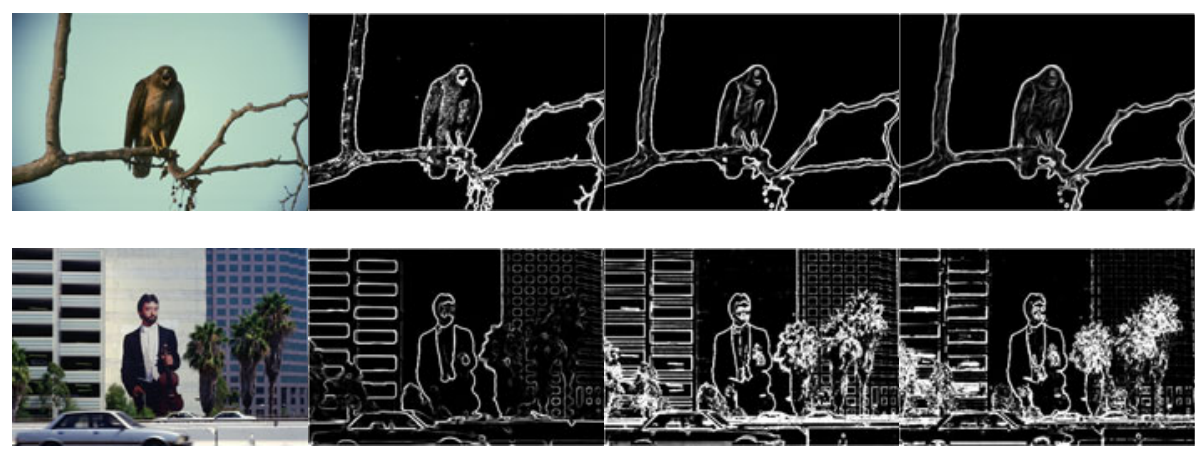

Fig. 2. Soft boundary maps obtained with images from the Berkeley Segmentation Dataset [12. The second column shows the results obtained from the grayscale version of the images; the third column contains the segmentation obtained from the RGB format images; and the fourth column presents the result obtained from the HSV format images. All the images were preprocessed with a $7 \times 7$ neighborhood size median filter and using 30 different evenly spaced $\sigma_{I}$ values in the intervals $[255,7650]$ for the grayscale case, $[1,30]$ for the RGB case and $[2,60]$ for the HSV case.

$\mathbf{x}_{0}=\mathbf{1}$. Searching for an explanation to this behavior, we noted that the adjacency matrix $\mathbf{W}$ has some characteristics that can be exploit to simplify our edge detection scheme. Our strongest supposition is that the adjacency matrix $\mathbf{W}$ can be approximated by $\mathbf{W} \approx \mathbf{I}+\mathbf{P}$, where $\mathbf{I}$ is an identity matrix of $N \times N$ and $\mathbf{P}$ is a matrix of $N \times N$ with all-one entries on the diagonals $n,-1,1$ and $n$, where the zero index corresponds to the main diagonal and the negative indexes correspond to the diagonals below the main diagonal and vice versa. Based on the former, we can take the liberty of approximate $\mathbf{D}$ by $\mathbf{D}^{*}=5 \mathbf{I}$.

Using both suppositions, we can approximate the normalized Laplacian by

$$
\mathbf{L}^{*}=\frac{4}{5} \mathbf{I}-\frac{1}{5} \mathbf{P} .
$$

Applying a shift with $s=2$ and deflation with $\lambda_{1}^{\prime}=-2$ and $\overline{\mathbf{u}}_{1}=\mathbf{u}_{1} /\left\|\mathbf{u}_{1}\right\|$, we obtain

$$
\mathbf{L}_{d}^{*}=\mathbf{L}_{s}^{*}+2 \overline{\mathbf{u}}_{1} \overline{\mathbf{u}}_{1}^{T}
$$

Is easy to verify that the second iteration of the power method using (12) will return a vector that is very close to a multiple of the first iteration, and the same will happen with the next iterations. Being like that, the first iteration of the power method is a good enough approximation of the second smallest eigenvector of $\mathbf{L}$. This means that we can get $\mathbf{u}_{2}$ as

$$
\mathbf{u}_{2}^{*}=-2 \mathbf{1}+2 \overline{\mathbf{u}}_{1} \overline{\mathbf{u}}_{1}^{T} \mathbf{1}
$$

This means that our edge detection method is no longer an iterative process, because (13) give us a closed form to obtain the segmentation. The segmentations obtained with (13) and the ones obtained with power method are visually 
undistinguishable. The former and the derivation of (13) are explained in detail in [8]. Our final version of the normalized cut based edge detection method is synthesized on Algorithm 1 .

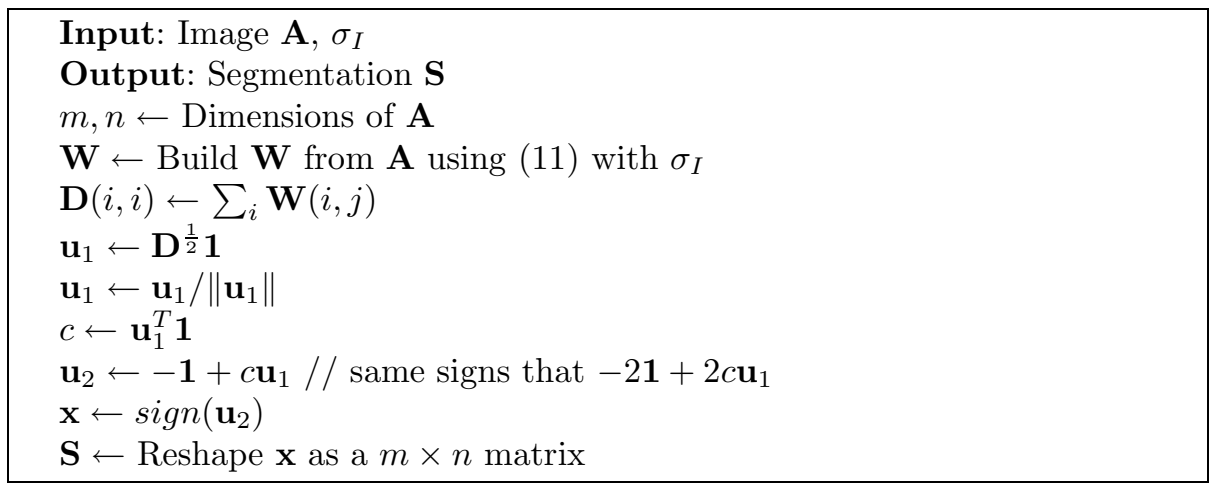

Algorithm 1. Normalized cut based edge detection

It is relevant to highlight some characteristics of the Algorithm 1, $\mathbf{W}$ is a symmetric pentadiagonal matrix with all-one elements on its main diagonal, which means that we only need to calculate two of the diagonals of $\mathbf{W}$. In fact, is not necessary to explicitly build $\mathbf{W}$, because is possible to obtain the entries of $\mathbf{D}$ directly. Moreover, $\mathbf{D}$ can be handled as a vector of dimension $N$. An appropriate implementation of this edge detection method can reduce the required storing space to a vector of $N$ elements. Also, the complexity of the algorithm is $O(N)$ with small constant.
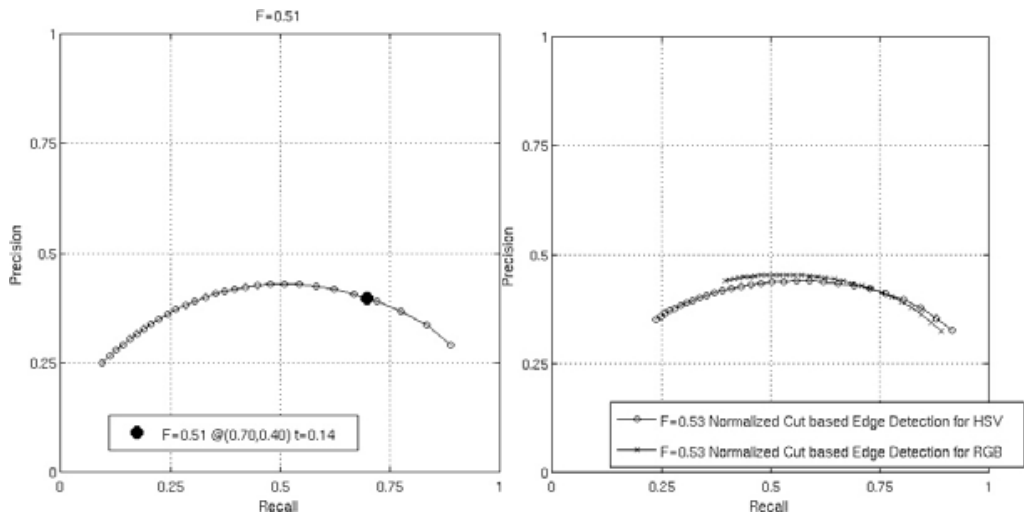

Fig. 3. Results obtained by our algorithms (grayscale on left and color on right) on the Berkeley Segmentation Benchmark using the same specs for the soft boundary maps as in the results of Fig. $2 F$ corresponds to the F-measure, which is the harmonic mean of precision and recall calculated at each level of the soft boundary map. The maximum F-measure value across an algorithm's precision-recall curve is reported as its summary statistic. 


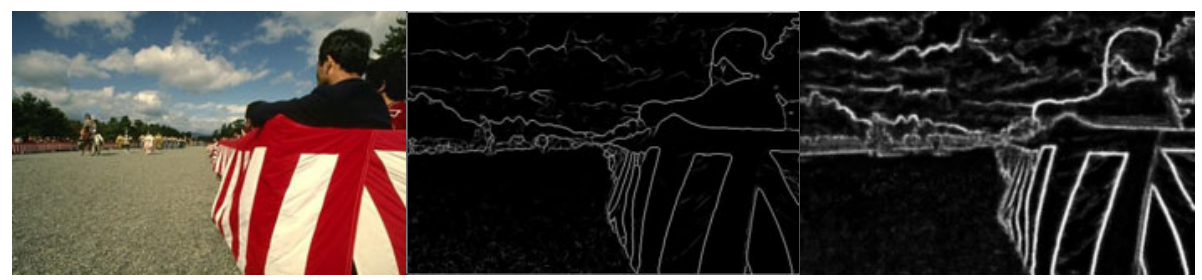

(a)

(b) $F=0.73$

(c) $F=0.85$

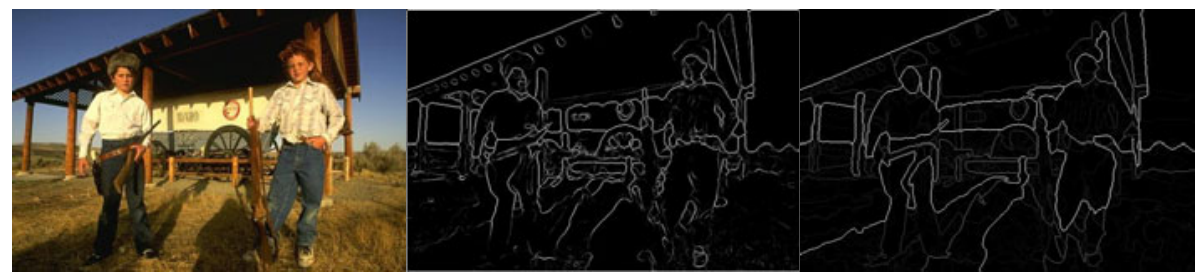

(d)

(e) $F=0.77$

(f) $F=0.88$

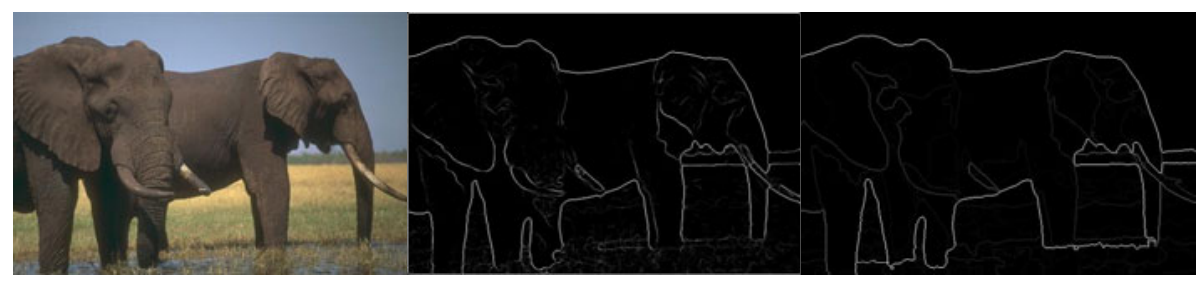

(g)

(h) $F=0.71$

(i) $F=0.86$

Fig. 4. Images from the BSD300 dataset segmented with the RGB version of the algorithm. Central columns shows our results using the specs indicated in Fig. 2 and the edge thinning algorithm included in MATLAB 11. Right column shows top results obtained with (c) boosted edge learning [13] and (f) and (i) with ultrametric contour maps 14. The $F$ measure is shown for every result (greater values means better qualification).

The segmentations obtained with this simplified method are graphically identical to the obtained with our first version of the method, but the simplified one has a remarkable superior performance. The results obtained on the Berkeley Segmentation Benchmark are shown on Fig. 3 . According to the obtained scores, our method is ranked tenth for the grayscale version and ninth for the RGB and HSV version on the list of reported edge detection algorithms. Figure 4 shows some comparisons of our method results with those of the best methods reported so far.

\section{Conclusions}

This paper presented a novel edge detection technique based on the original segmentation scheme introduced by Shi-Malik. The method was developed mainly 
by moderately modifying the function that relates the pixels and by considering only the relation among the pixels on a neighborhood of radius one. Using a very simple approximation of the adjacency matrix lead us to obtain the edge detection with a very simple closed form, basically a sum of vectors. The most remarkable characteristic of the method is its simplicity, which translates in economy of computational resources.

The method was capable of work with images on color and grayscale images. Overall results are on the level of those of the gradient methods, but ours have a high variability. We considered that this notorious variation of scores between images is related with the textures, since the best results are obtained with images containing simple textures.

\section{References}

1. Wu, Z., Leahy, R.: An optimal graph theoretic approach to data clustering: Theory and its application to image segmentation. IEEE Transactions on Pattern Analysis and Machine Intelligence 15(11) (1993)

2. Shi, J., Malik, J.: Normalized cuts and image segmentation. IEEE Transactions on Pattern Analysis and Machine Intelligence 22(8) (2000)

3. Avalos, V.: Segmentación de imágenes usando técnicas espectrales de grafos (2007)

4. Golub, G., Van-Loan, C.: Matrix Computations, 3rd edn. John Hopkins Press, Baltimore (1996)

5. Pothen, A., Simon, H.D., Liou, K.P.: Partitioning sparse matrices with eigenvectors of graphs. SIAM Journal on Matrix Analysis and Applications 11(3), 430-450 (1990)

6. Langville, A.N., Meyer, C.D.: Google's PageRank and Beyond: The Science of Search Engine Rankings, pp. 40-41. Princeton University Press, Princeton (2006)

7. Chung, F.: Spectral Graph Theory. Number 92 in CBMS Regional Conference Series in Mathematics. American Mathematical Society, Providence (1997)

8. Madrid, H., Barrientos, M.: Detección de bordes basada en corte normalizado. In: Latin American Conference on Networked and Electronic Media (2010)

9. Arce, G.R.: Nonlinear signal processing: a statistical approach. Wiley, Chichester (2005)

10. Lindeberg, T.: Edge detection and ridge detection with automatic scale selection. International Journal of Computer Vision 30(2), 117-154 (1996)

11. MathWorks: Morphological operations on binary images (2011)

12. Martin, D., Fowlkes, C., Tal, D., Malik, J.: A database of human segmented natural images and its application to evaluating segmentation algorithms and measuring ecological statistics. In: Proc. 8th Int'l Conf. Computer Vision, vol. 2, pp. 416-423 (2001)

13. Dollar, P., Tu, Z., Belongie, S.: Supervised learning of edges and object boundaries. In: IEEE Computer Vision and Pattern Recognition, CVPR 2006 (2006)

14. Arbeláez, P.: Boundary extraction in natural images using ultrametric contour maps. Technical report, Université Paris Dauphin (2006) 